\title{
Approaches to Improving Nursing Handoffs in Surgical Wards
}

\author{
Katherine S. Blondon ${ }^{1}$, Frederic Ehrler ${ }^{2}$, Sandrine Le Godais ${ }^{3}$, Jean-Yves Wojtasikiewicz ${ }^{3}$, \\ Charline Couderc ${ }^{3}$
}

${ }^{1}$ Medical Directorate, University Hospitals of Geneva, Geneva, Switzerland

${ }^{2}$ Division of Medical Information Science, University Hospitals of Geneva, Geneva, Switzerland

${ }^{3}$ Department of Surgery, University Hospitals of Geneva, Geneva, Switzerland

Email: ${ }^{\star}$ Katherine.Blondon@hcuge.ch

How to cite this paper: Blondon, K.S., Ehrler, F., Le Godais, S., Wojtasikiewicz, J.-Y. and Couderc, C. (2017) Approaches to Improving Nursing Handoffs in Surgical Wards. Open Journal of Nursing, 7, 10341043.

https://doi.org/10.4236/ojn.2017.79075

Received: July 24, 2017

Accepted: September 15, 2017

Published: September 18, 2017

Copyright (๑) 2017 by authors and Scientific Research Publishing Inc. This work is licensed under the Creative Commons Attribution International License (CC BY 4.0).

http://creativecommons.org/licenses/by/4.0/ c) (i) Open Access

\begin{abstract}
Objective: To propose approaches to improve nursing handoffs for surgical patients, including standardization. Background: Handoffs, or the transfer of accountability and patient information, can generate potential risks for patient safety. Standardization has been proposed to help improve handoffs. Methods: After observing 333 nursing handoffs in the surgical wards of our institution, we conducted a thematic content analysis, comparing and contrasting the observations. Results: Handoff processes, including the use of support tools, varied among the observations. Common themes in the handoff content suggested possibilities of standardization. About half of the 51 interruptions occurring during the observed handoffs were by healthcare professionals. Conclusions: Standardization to improve handoffs should address both the content and the process. Interruptions were common and should be avoided whenever possible. Future studies should also consider the use of mobile applications to support handoffs and clinical documentation.
\end{abstract}

\section{Keywords}

Nursing Handoffs, Handover, Nursing Sign-Out, Shift Report, Bedside Handoff

\section{Introduction}

Continuous 24/24 h patient care in hospital wards requires handoffs between shifts. Handoffs are the transfer of accountability and patient information among care providers [1]. They are moments of potential risk for safety in patient care, with possible adverse events [2]. In prior studies on patient safety and preventable adverse events, about $60 \%$ of the events are associated with commu- 
nication issues, in particular during handoffs [3]. Improving handoffs has recently become an important focus for safer patient care. Handoffs face several challenges, such as quality of content, time constraints, wide variability between wards and interruptions. Standardization of the handoff process, often with the use of mnemonics, has been effective to improve the quality of the content [4] [5]. Bedside handoffs are another proposed approach to improve the handoff process, in particular for patient safety [6] [7].

The format of nursing handoffs in the 11 surgical wards of our hospital varies according to the time of day and subsequent staffing. The wards have specialties: urology, transplantation, cardiovascular surgery (each one ward), general surgery ( 4 wards) and orthopedic surgery ( 4 wards). Each ward requires three or four nurses during the morning shift, two or three nurses in the afternoon with a mid-afternoon handoff, and one or two nurses during the night shift. Nurse assistants, present throughout the 24-hour period, also attend the handoffs. They help with many of the tasks at the bedside, but are not involved for medication, or tests such as blood work or electrocardiograms. In our teaching hospital, surgery wards have up to 20 patients, and handoffs between doctors and nurses occur separately. The duration of handoffs ranges from $15 \mathrm{~min}$ at the start of the day (from the night shift team), to $60 \mathrm{~min}$ during the mid-day handoff.

The aim of this study is to observe the current nursing handoff process in surgical wards, and to explore approaches for improvement, in particular for standardization, using a qualitative analysis.

\section{Methods}

This is an observational qualitative study of nursing handoffs. Three investigators (head nurses) observed morning and afternoon nursing handoffs in the different surgical wards. To report the content of the handoffs, they first developed a paper-based observation tool (Figure 1): based on preliminary observations of the handoff process, the investigators iteratively compared and extracted common themes covered during the handoff process, such as patient identity, allergies, and medications. This tool allowed the observers to code their observations of the handoffs in real time.

Observers assessed whether these themes were simply mentioned or discussed in detail, and whether the discussed elements were pertinent. They also reported the type of support tools used and potential barriers for handoffs. After 2 weeks of observations, the investigators summarized the coded observations from the paper-based tool, comparing and contrasting the results from the different types of surgical wards. Based on the results, we propose approaches to improve the handoff process.

\section{Results}

\subsection{General Observations}

The three investigators observed a total of 333 handoffs during the two-week 


\begin{tabular}{|c|c|c|c|c|}
\hline Theme & Mentioned & $\begin{array}{l}\text { Detailed information } \\
\text { provided }\end{array}$ & Pertinence & Comments \\
\hline \multicolumn{5}{|l|}{ Prior knowledge of the patient } \\
\hline \multicolumn{5}{|l|}{\begin{tabular}{|l} 
Patient name \\
- DOB
\end{tabular}} \\
\hline \multicolumn{5}{|l|}{ Patient room number and position } \\
\hline $\begin{array}{l}\text { Diagnosis } \\
\text { Medical history } \\
\text { Surgical procedure } \\
\text { Post-operation day }\end{array}$ & & & & \\
\hline \multicolumn{5}{|l|}{ Infection control information } \\
\hline \multicolumn{5}{|l|}{ Allergies } \\
\hline \multirow{2}{*}{\multicolumn{5}{|c|}{ Diet/Fasting }} \\
\hline & & & & Equipments \\
\hline \multicolumn{4}{|l|}{$\begin{array}{l}\text { Medication } \\
\text { - Premedication }\end{array}$} & \\
\hline $\begin{array}{l}\text { Peridural/block anesthesia } \\
\text { - level } \\
\text { - effectiveness }\end{array}$ & & & & \\
\hline \multicolumn{5}{|l|}{ Wound \& skin } \\
\hline \multirow{2}{*}{\multicolumn{5}{|c|}{\begin{tabular}{|l} 
Lab tests of the day \\
Transfusion
\end{tabular}}} \\
\hline & & & & \\
\hline \multicolumn{5}{|l|}{\begin{tabular}{|l} 
Transfusion \\
Bowel movements
\end{tabular}} \\
\hline \multicolumn{5}{|l|}{ Autonomy } \\
\hline \multicolumn{5}{|l|}{ Ins \& Outs } \\
\hline $\begin{array}{l}\text { Other information } \\
\text { - Problems of the day } \\
\text { - Appointments, tests } \\
\text { - Results, reports }\end{array}$ & & & & \\
\hline \multicolumn{5}{|l|}{ Monitoring } \\
\hline \multicolumn{5}{|l|}{ Transfer/discharge/paperwork } \\
\hline \multicolumn{5}{|l|}{ Social situation } \\
\hline $\begin{array}{l}\text { Issues related to workload } \\
\text { Issues related to collaboration with } \\
\text { doctors } \\
\text { Issues related to patient/family }\end{array}$ & & & & \\
\hline Interruptions & & & & \\
\hline
\end{tabular}

Figure 1. Paper-based observation tool.

period. We present the findings from the various handoff features, and then describe the general process and barriers for handoffs.

\subsection{Handoff Content}

Handoffs typically always began the same way with patient identification (name and age or year of birth). The content then differed by type of surgical ward. The nurses generally used the problem list to structure their narrative with details of the management plan, equipment and comorbidities. Pain management was also a dominant theme, but the intensity of the pain on the visual-analog scale was not often reported. The nurses described the patient's diagnoses and interventions, and provided the admission date or days since surgery. The past medical history was included if the diagnoses were considered pertinent for patient care during the stay or at discharge (i.e., diabetes, or dementia). Certain types of medications such as antibiotics, anticoagulants and insulin were systematically reported in detail, whereas other medications were not always mentioned. Postoperative prescriptions included treatments and surveillance orders. Nurses in general surgery and the other wards then discussed patient equipment. In orthopedic nursing handoffs, the equipment was associated with medications, patient care or monitoring. Some handoffs also discussed discharge plans, monitoring or pressure ulcers. Table 1 provides a comparison of the top 10 handoff 
Table 1. Top ten handoff themes discussed by type of surgical ward.

\begin{tabular}{lccc}
\hline & Orthopedics & General surgery & Other surgical wards ${ }^{*}$ \\
\hline 1 & Patient ID & Patient ID & Patient ID \\
2 & Surgery & Medication & Surveillance \\
3 & Days post-op & Equipment & Surgery \\
4 & Medication & Surgery & Age / Days post-op \\
5 & Pain & Age & Equipment \\
6 & Discharge & Diagnoses & Past medical history \\
7 & Equipment & Days post-op & Discharge \\
8 & Daily concerns & Past medical history & Skin condition \\
9 & Age & Pain & Diagnoses \\
10 & Past medical history & Skin condition & Discharge \\
\hline
\end{tabular}

*Urology, transplantation and cardiovascular surgery.

themes discussed by type of surgical ward.

\subsection{Handoff Process and Use of Support Tools}

Overall, handoff duration ranged from about 1 to 3.5 minutes per patient. The duration of the handoffs varied according to certain parameters: time of day, type of surgical ward, individual handoff style, training of the receiver, interruptions and prior knowledge of the patient. Morning handoffs tended to be shorter than the afternoon handoffs.

Nurses' handoffs have two components: a verbal, in-person component and a written component in the nursing notes of the EHR. For the verbal component, nurses used several types of supportive tools.

Handwritten notes: Use of personal notes was common. Nurses took notes during the handoffs on blank paper, a printout of the patient list, or a Word or Excel template with patient identity, room number and tasks. They kept these notes in their pockets, and updated them throughout the shift with reminders, such as to fax a prescription. When working consecutive days, nurses sometimes re-used their personal notes, simply crossing off the discharged patients, and adding the new ones.

Printouts of nursing tasks: All nurses had a printout of the task list for each of their patients, with the medication list and other patient care information (i.e., assistance for meals or mobilization). These printouts were generally printed by the handoff giver just prior to the handoff, to have updated medical prescriptions at the beginning of each shift. Some nurses also annotated this print out during the handoffs. Although all wards printed these task lists, they were not all used during the handoffs. Most of the time, nurses documented the main diagnoses, code status, and main concerns for the shift, which are not in the printout.

Postoperative prescription forms: anesthetists made the post operative pre- 
scriptions on paper documents that are also used in the recovery room. These postoperative medical orders are not transcribed in the EHR for the wards, but remain valid until the ward physician prescribes new orders, or at most up to $24 \mathrm{~h}$ after arriving in a ward.

Patient information board: Handoffs took place in nursing offices, which provided an access to the patient information board and to three desktop computers. The patient information board provides an outlay of the ward, with patient and nursing team names, as well as surgical specialty. It also has discharge indications and monitoring requirements, which gives the team an overview of the patient workload. After the handoffs, nurses had team discussions about logistics or complex situations.

EHR dashboard: Another supportive tool used for handoffs was the patient dashboard in the EHR. It provides an overview of a patient's administrative data (name, age or year of birth), past medical history and current diagnoses, vital signs and trends as well as the current medical equipment.

The EHR dashboard contains several sections with different types of patient information, which can support handoffs (Figure 2). 1) Patient identity with the age helps avoid confusion with names that might be similar. 2) This section contains general information about the patient: the reason of admission, past medical history as well as information about the social support and environment of the patient. 3) Section 3 presents a visual overview of the patient's current equipment. 4) A graphical overview of the patient's vital signs with the recent values and trends, as well as the intervention date(s) can be found in Section 4. Clicking on this section opens up the global graphic view of the patient's charts, which includes weight, pain scores and administered medications.

Nurses did not rely on their EHR nursing chart notes for handoffs, mainly because they were often only completed after the verbal handoff.

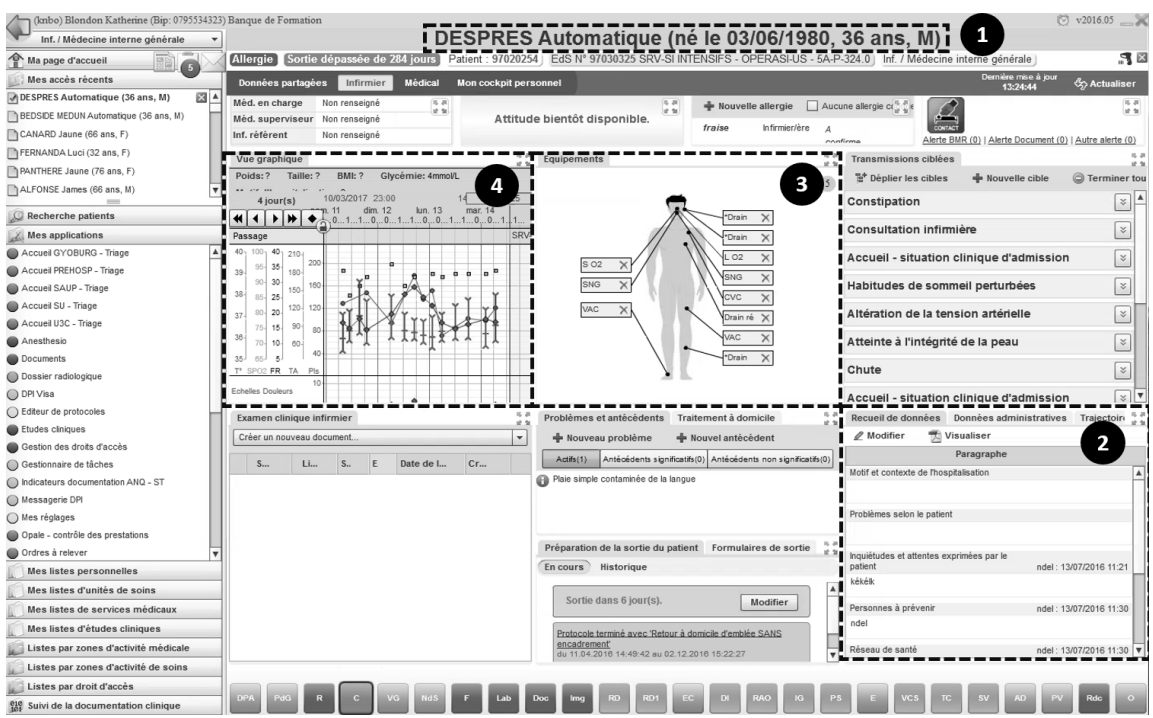

Figure 2. Using the EHR dashboard to support handoffs: (1) patient identity, (2) general medical information about the patient, (3) patient equipment, (4) Vital signs. 
The use of the different supportive tools by type of ward is presented in Table 2. In the orthopedics and general surgery wards, nurses relied mainly on their personal notes and on the board in the nursing office. Nurses in the skin surgery wards, however, tended to use the patient dashboard in the EHR as well as their notes and the board. When medications were discussed, they were often simply listed, with little additional information.

Nurses wrote down vital signs on their notes before entering the data into the EHR at a later time. These notes were also used to give the handoff at the end of a shift.

\subsection{Barriers to Handoffs}

Several barriers to handoffs were identified during the observations. The main barrier was the frequent interruptions. During the observation period, handoffs were interrupted 51 times. Twenty-six interruptions were by colleague nurses, physicians, physiotherapists, or dieticians, and nine were phone calls. Another nine interruptions were for patient transports for planned exams or interventions. Seven interruptions were questions from the patients' visitors.

Another barrier for handoffs and patient care was the presence of substitutenurses. These nurses are not part of the usual ward teams. Although they are experienced and competent, they may not be familiar with certain equipment, medications, or with certain protocols that are specific to each ward.

Finally, the use of supportive tools for handoffs was more difficult when several people took part in a handoff, such as during morning handoffs, due to screen sizes of laptops or even desktop computers. Moreover, reading the printouts and note-taking occupied the handoff receiver most of the time, and sometimes slowed the flow of the verbal handoffs.

\subsection{Other Observations}

Observations about the handoff processes revealed other opportunities for improvement. First, there was a tendency to use informal, non-medical language during the handoffs, rather than medical terms (i.e., "he was burning up" rather than "he had a fever"). Although this approach made the tone more casual, precision of the handoff information was sometimes affected.

Second, the location of the handoffs varied among the wards. Most handoffs took place in the nurses' office, some in the corridor in front of the patient

Table 2. Comparison of supportive tools used for handoffs in different wards.

\begin{tabular}{cccc}
\hline & Orthopedic wards & $\begin{array}{c}\text { General surgery and } \\
\text { transplantation wards }\end{array}$ & Other wards \\
\hline EHR dashboard & $0.7 \%$ & $4.25 \%$ & $41.17 \%$ \\
Nursing tasks list & $2.12 \%$ & $3.54 \%$ & $19.6 \%$ \\
Postoperative prescriptions & $1.41 \%$ & $2.83 \%$ & $5.88 \%$ \\
Personal notes + information board & $95.77 \%$ & $89.38 \%$ & $33.34 \%$ \\
\hline
\end{tabular}


rooms, some in the break room, and a minority took place at the bedside. Finally, handoffs were often delayed, either due to individual tardiness or to on-going team discussions such as scheduling issues.

\section{Discussion}

Based on the 333 observations in 11 surgical wards of our institution, we described the processes, including the use of support tools and content of handoffs between the teams and content of handoffs. This observation revealed a high variability that has been reported in the literature [8] [9]. A range of factors such as established routines, available resources, and differences in clinical needs across the surgical subspecialties contribute to this variability.

One of the key recommendations for improvement in the handoff literature is standardization, both of the process and the content. In the next part of the discussion, we describe recommendations, based on our observations.

\subsection{Standardizing the Handoff Process}

Documentation in the EHR was often delayed, with potential transcription errors from the initial annotation on personal notes. Progress notes or other task-related annotations, for example, were often written up at the end of a shift, after giving the handoff. This data collection was delayed until the nurses got back to the nursing office, and logged into the EHR. This delay engenders a potential risk of omitting elements during the handoff and can be shortened with the use of computer on wheels (COWs) at the bedside. Handoffs improvement can help avoid omissions and decrease errors [4] [10].

Our recommendations to standardize the handoff process are to begin the handoffs with a brief $(<10 \mathrm{~min})$ overview of the patients in the ward in the nursing office, with patient name and birth date, type of intervention with indication of postoperative day, daily objective. This overview also should include planned admissions and discharges of the day. The detailed patient handoff is conducted at the bedside, using the EHR dashboard as support to reduce errors [11].

Nursing assistants can contribute to handoffs, and also benefit from receiving information about the patients for their shifts. Although patient confidentiality and seating arrangements are better addressed in the nurses' office, bedside handoffs allow patients to be involved in their care, and promote higher patient safety [12] [13].

\subsection{Standardizing the Handoff Content}

Standardizing the handoff process has been widely explored in the handoff literature, particularly with the development of mnemonics [5] [6] [7] [14]. Nurses tended to have a common structure for verbal handoffs, as they discussed many common themes for their patients. Using a common structure can help the receiver create her own mental model for each patient. In addition to the use of 
many common themes among the different wards, the handoffs in our study did seem to follow a general outline with the following themes: 1) identification of the patient and code status, 2) reason for admission, 3) surgical intervention, 4) relevant medical history, 5) current problem list and treatments, 6) daily concerns and 7) discharge planning.

\subsection{Standardizing the Use of Support Tools}

Our observations showed varied support tool use when receiving a handoff, and when collecting these facts during the shift. This variability was due to two main factors: first, head nurses' recommendations played an important role in EHR use to support handoffs or not (no institutional recommendation) Second, usability and efficiency of the EHR also affect the teams' preferences for this process. Connectivity issues with Wi-Fi for the COWs slowed the navigation between patient charts and discouraged some teams from using this support. Some teams also reported that the design of the EHR did not support the way they conducted their handoffs, and that it took too long to navigate to the required information in the EHR.

We observed high use of personal notes and low use of EHR during handoffs. Printouts were not used systematically during handoffs. Most of the time, nurses documented the main diagnoses, code status, and main concerns for the shift. Although writing down key points can help improve the retention of information, it is also time-consuming, tedious, and a potential source of error. Errors can arrive from transcriptions, miscomprehension, or from the distraction of having to take notes while the colleague continues talking. Beyond the handoff itself, our observations showed that using printouts of the daily task listduring shifts was common to all wards. Prior literature on printouts emphasizes the risk of potential errors when the data is modified in the HER [15].

The dashboard section of our EHR can support and offs, in particular to help standardize the content of the handoff. COWs also provide easy access to the EHR at the bedside. Using the dashboard as support, particularly at the bedside, can help the nurses to improve the precision of the handoff content. It also enables nurses to respond to patient questions at the bedside, and may also help anticipate nursing care.

Another approach for a support tool would be to provide a summary of the task-related annotations, which can be consulted during the handoff to ensure that all relevant topics have been discussed. This type of support could be provided in a mobile app, which could provide access to the task list. The additional advantage of a mobile app in this situation over a COW is its more ubiquitous availability, since COWs cannot be used in all environments (i.e., pathogen-free environments), for example. Reports of mobile tools to support bedside care are appearing in the literature [16] [17], with rapid adoption and high user satisfaction.

There are limitations to our study. First, observers were not familiar with the patient cases, and were therefore not able to judge the pertinence of the handoff 
content. Second, the presence of observers may influence the performance. The charge nurses who are often present during handoffs reported that this effect seemed rather modest over all the observations. Finally, our study focuses on handoff processes in the surgical wards of our institution, which may limit its generalizability to other surgical settings or to other medical specialties.

\section{Conclusions}

Based on our observations, we propose approaches to standardize both the process and content of handoffs. The standardized morning handoff process should occur as a team, based on the EHR synoptic view of all the patients of the ward. The night nurse will present the patients, focusing particularly on recent events and concerns, and concerns or aims for the day. Based on the EHR synopsis of the ward, we propose that the night nurse presents the room number, patient ID, reason of admission and daily concerns. For the mid-afternoon handoff, we propose to begin as a team with an initial 10-min overview in the nurse office, followed by bedside handoffs in smaller teams (nurses and nursing assistants).

Moving the handoffs to the bedside allows the patient and family to be involved, and can help improve comprehension of planned care. It is also an opportunity to introduce the on-coming nurse to the patients. Although bedside handoffs have many benefits, confidential issues may be delicate to address in the presence of other patients in the room, and visitors need to be asked to wait outside. Computers-on-wheels (COWs) allow EHR access during bedside handoffs, and can provide complementary support to personal notes for most of the handoff content. After the bedside handoff, nurses can then briefly discuss how to distribute the tasks before the morning shift workers leave.

\section{References}

[1] Solet, D.J., Norvell, J.M., Rutan, G.H. and Frankel, R.M. (2005) Lost in Translation: Challenges and Opportunities in Physician-to-Physician Communication during Patient Handoffs. Academic Medicine: Journal of the Association of American Medical Colleges, 80, 1094-1099. https://doi.org/10.1097/00001888-200512000-00005

[2] Starmer, A.J., Sectish, T.C., Simon, D.W., Keohane, C., McSweeney, M.E., Chung, E.Y., et al. (2013) Rates of Medical Errors and Preventable Adverse Events among Hospitalized Children Following Implementation of a Resident Handoff Bundle. JAMA: The Journal of the American Medical Association, 310, 2262-2270. https://doi.org/10.1001/jama.2013.281961

[3] Commission, T.J. (2013) Sentinel Event Data-Root Causes by Event Type 2013. http://www.jointcommission.org/Sentinel_Event_Statistics/

[4] Arora, V. and Johnson, J. (2006) A Model for Building a Standardized Hand-Off Protocol. Joint Commission Journal on Quality and Patient Safety/Joint Commission Resources, 32, 646-655. https://doi.org/10.1016/S1553-7250(06)32084-3

[5] Nasarwanji, M.F., Badir, A. and Gurses, A.P. (2016) Standardizing Handoff Communication: Content Analysis of 27 Handoff Mnemonics. Journal of Nursing Care 
Quality, 31, 238-244. https://doi.org/10.1097/NCQ.0000000000000174

[6] Taylor, J.S. (2015) Improving Patient Safety and Satisfaction with Standardized Bedside Handoff and Walking Rounds. Clinical Journal of Oncology Nursing, 19, 414-416. https://doi.org/10.1188/15.CJON.414-416

[7] Anderson, J., Malone, L., Shanahan, K. and Manning, J. (2015) Nursing Bedside Clinical Handover-An Integrated Review of Issues and Tools. Journal of Clinical Nursing, 24, 662-671. https://doi.org/10.1111/jocn.12706

[8] Ilan, R., LeBaron, C.D., Christianson, M.K., Heyland, D.K., Day, A. and Cohen, M.D. (2012) Handover Patterns: An Observational Study of Critical Care Physicians. BMC Health Services Research, 12, 11. https://doi.org/10.1186/1472-6963-12-11

[9] Riesenberg, L.A., Leitzsch, J. and Cunningham, J.M. (2010) Nursing Handoffs: A Systematic Review of the Literature. The American Journal of Nursing, 110, 24-34. https://doi.org/10.1097/01.NAJ.0000370154.79857.09

[10] Starmer, A.J., Landrigan, C.P. and Group, I.P.S. (2015) Changes in Medical Errors with a Handoff Program. The New England Journal of Medicine, 372, 490-491. https://doi.org/10.1056/NEJMc1414788

[11] Johnson, M., Sanchez, P. and Zheng, C. (2016) Reducing Patient Clinical Management Errors Using Structured Content and Electronic Nursing Handover. Journal of Nursing Care Quality, 31, 245-253. https://doi.org/10.1097/NCQ.0000000000000167

[12] Maxson, P.M., Derby, K.M., Wrobleski, D.M. and Foss, D.M. (2012) Bedside Nurse-to-Nurse Handoff Promotes Patient Safety. Medsurg Nursing, 21, 140-144.

[13] Mardis, T., Mardis, M., Davis, J., Justice, E.M., Riley Holdinsky, S., Donnelly, J., et al. (2016) Bedside Shift-to-Shift Handoffs: A Systematic Review of the Literature. Journal of Nursing Care Quality, 31, 54-60. https://doi.org/10.1097/NCQ.0000000000000142

[14] Riesenberg, L.A., Leitzsch, J. and Little, B.W. (2009) Systematic Review of Handoff Mnemonics Literature. American Journal of Medical Quality: The Official Journal of the American College of Medical Quality, 24, 196-204. https://doi.org/10.1177/1062860609332512

[15] Rosenbluth, G., Jacolbia, R., Milev, D. and Auerbach, A.D. (2016) Half-Life of a Printed Handoff Document. BMJ Quality \& Safety, 25, 324-328. https://doi.org/10.1136/bmjqs-2015-004585

[16] Motulsky, A., Wong, J., Cordeau, J.P., Pomalaza, J., Barkun, J. and Tamblyn, R. (2016) Using Mobile Devices for Inpatient Rounding and Handoffs: An Innovative Application Developed and Rapidly Adopted by Clinicians in a Pediatric Hospital. Journal of the American Medical Informatics Association, 24, e69-e78. https://doi.org/10.1093/jamia/ocw107

[17] Tang, C. and Carpendale, S. (2008) Evaluating the Deployment of a Mobile Technology in a Hospital Ward. Proceedings of the 2008 ACM Conference on Computer Supported Cooperative Work, San Diego, 8-12 November 2008, 205-214. 
Submit or recommend next manuscript to SCIRP and we will provide best service for you:

Accepting pre-submission inquiries through Email, Facebook, LinkedIn, Twitter, etc. A wide selection of journals (inclusive of 9 subjects, more than 200 journals)

Providing 24-hour high-quality service

User-friendly online submission system

Fair and swift peer-review system

Efficient typesetting and proofreading procedure

Display of the result of downloads and visits, as well as the number of cited articles Maximum dissemination of your research work

Submit your manuscript at: http://papersubmission.scirp.org/

Or contact ojn@scirp.org 